\title{
La planeación del emprendimiento empresarial. Un enfoque a partir de la economía política
}

\section{The planning of entrepreneurial entrepreneurship. An approach based on political economy}

José Fabián Fonseca Vásconez. ${ }^{1}$, Norma Yolanda Villacis Venegas. ${ }^{2}$, Norma del Rocio Toledo Castillo. ${ }^{3}$, German Patricio Torres Guananga. ${ }^{4} \&$ Marco Vinicio Salazar Tenelanda ${ }^{5}$

\begin{abstract}
.
DOI: https://doi.org/10.33262/cienciadigital.v3i2.6.576

The creation of a company in a world without commercial borders governed by competitiveness like the one we are currently experiencing is a complex process, therefore, those who want to undertake a productive activity fail to consider that it is enough their entrepreneurial spirit to put their idea to work in the market assuming the success or risk of failure as normal for an entrepreneur, in this reason the present study aims to demonstrate that it is possible to prevent the forces of supply and demand market destroy a nascent productive activity, for which purpose is proposed as planning entrepreneurship from the perspective of political economy, in this area the interaction of its theoretical-methodological livelihoods is a guide for business research and suggests the theoretical basis for entrepreneurs from an area of knowledge of the social sciences; whose conclusions open future studies on the importance of promoting cooperation on competitiveness to reduce hazards and promote sustained economic

1 Universidad Técnica Estatal de Quevedo, Facultad de Ciencias empresariales, Quevedo, Ecuador,

2 Escuela Superior Politécnica de Chimborazo, Facultad de Administración de Empresas, Riobamba, Ecuador, norma.villacis@espoch.edu.ec

3 Escuela Superior Politécnica de Chimborazo, Facultad de Administración de Empresas, Riobamba, Ecuador, norma.toledo@espoch.edu.ec

4 Escuela Superior Politécnica de Chimborazo, Facultad de Administración de Empresas, Riobamba, Ecuador, g_torres@espoch.edu.ec

5 Escuela Superior Politécnica de Chimborazo, Facultad de Administración de empresas, Riobamba, Ecuador, salazarmarco1977@gmail.com
\end{abstract} jfonsecav@uteq.edu.ec 
growth since the creation of a company, appears as an alternative to the serious problem of unemployment that cross the nations.

Keywords: Planning, political economy, entrepreneurship, competitiveness, coopetition.

\section{Resumen.}

La creación de una empresa en un mundo sin fronteras comerciales regido por la competitividad como el que actualmente vivimos es un proceso complejo, por tanto, quienes quieren emprender una actividad productiva fracasan al considerar que basta su espíritu empresarial para poner su idea a funcionar en el mercado asumiendo el éxito o el riesgo de fracaso como algo normal para un emprendedor, en tal razón el presente estudio tiene como objetivo demostrar que es posible evitar que las fuerzas de mercado oferta y demanda destruyan una actividad productiva naciente, para lo cual se propone como planificar el emprendimiento desde la visión de la economía política, en este ámbito la interacción de sus sustentos teóricos-metodológicos constituye una guía para las investigaciones empresariales y sugiere la base teórica para emprendedores a partir de una área de conocimiento de las ciencias sociales; cuyas conclusiones dan apertura a futuros estudios sobre la importancia de promover coopetencia sobre la competitividad para reducir peligros y fomentar el crecimiento económico sostenido ya que la creación de una empresa, aparece como una alternativa al grave problema del desempleo que atraviesan las naciones.

Palabras claves: Planificación, economía política, emprendimiento, competitividad, competencia.

\section{Introducción}

Uno de los grandes retos para la sociedad de este nuevo milenio, sin duda, es impulsar el desarrollo de las estructuras sociales, institucionales, ambientales y económicas del ámbito local, nacional y mundial, frente al cada vez más caótico proceso de globalización que orienta al ser humano a la competitividad y no por la cooperación, la equidad, la igualdad y la solidaridad; considerando a la naturaleza como un objeto de derecho y no como un sujeto de derecho; destruyendo al mismo tiempo débiles sistemas empresariales, sin considerar los efectos multiplicadores negativos que genera al del entorno socioeconómico al territorio donde la empresa ejerce su actividad.

Según la medición que realiza la Escuela de Negocios de la Espol para el Global Entrepreneurship Monitor, que mide el emprendimiento en 66 países que representan el $69,2 \%$ de la población mundial, Ecuador se posiciona por sexto año consecutivo como líder en la región.

El estudio muestra que cerca de 3 millones de adultos en el país pusieron en marcha un emprendimiento naciente, lo que representa el 29,6\% de la población entre 18 y 64 años, sin embargo, determinándose que en la República del Ecuador el emprendedor ecuatoriano tiene 
en promedio 36 años, su negocio esta dirigido directamente al consumidor, el 48,8\% proviene de hogares con ingreso mensual entre $\$ 375$ y $\$ 750$, y no cuenta con estudios de tercer nivel. Destacándose, además, el $33.4 \%$ tiene un empleo adicional a su negocio, el $50.2 \%$ es empleado de su propio emprendimiento.

En este mismo ámbito, la tasa de los que emprenden por oportunidad supera a la de quienes lo hace por necesidad (57.31\% vs. $42.33 \%$ ); sin embargo, no se ha logrado genera soluciones a las contradicciones derivadas de los problemas procedentes de las relaciones de producción, ya que en la actualidad la curva de emprendimiento ha decaído durante el primer semestre del 2019; destacándose que el 70\% de los emprendimientos fracasan.

Por tal razón, para transformar el estado de cosas antes referido, es necesario desentrañar las leyes que presiden el desarrollo, es decir, las relaciones de producción entre los hombres, en el proceso de producción de los bienes materiales, para comprender la correlación de las fuerzas productivas y las relaciones de producción; en este sentido, la Economía Política es fundamentada como ciencia social que establece la base teórica para el diseño de las políticas económicas y sus fenómenos, por lo cual se plantea como objetivo central planificar el emprendimiento empresarial a partir de la economía política.

En este ámbito, los autores delinean su investigación a partir de las funciones de la economía política; Marx en el Capital, (Marx, s/f) hace explicita la importancia del estudio de esta ciencia a través de sus funciones cognoscitiva, metodológica, ideológica y práctica; cuyas interrelaciones permiten obtener soluciones a las contradicciones derivadas de los problemas procedentes de las relaciones de producción.

Si extraemos de la función cognitiva en el objeto de estudio, los términos básicos conocimiento y emprendimiento empresarial, pasamos a una fase superior y comenzamos a hablar de emprendimientos inteligentes capaces de crear su propio compendio de conocimientos económicos. Estamos, por tanto, en la búsqueda de rentabilidad con un conocimiento adquirido, generado, identificado y transmitido para crear ventajas económicas de producciones locales, estructuras sostenibles de redes productivas establecidas en cadenas de suministros integradas con tecnología local innovadora, todo ello reflejado en una cultura planificada del emprendimiento.

Una de las categorías de este estudio es la sostenibilidad, la cual no es factible solo en el marco teórico, sino que también debe tener su comprobación práctica, por lo que: ¿cómo lograr un emprendimiento empresarial sostenible? Pues la principal respuesta a esta interrogante está en la práctica, la cual permitirá soportar las funciones cognitivas, metodológicas e ideológicas y viceversa.

Es decir, al analizar las sociedades de hoy, se podría concluir que su desarrollo se debe a que han implementado el fenómeno del emprendimiento con diversas características de motivaciones de afiliación, logro y poder como directrices para lograr un desempeño eficaz dentro de sus empresas y contribuir con su crecimiento. (Rodríguez, 2009) 
Por tanto, es fundamental comprender que las funciones de la Economía Política no solo se interrelacionan, además tienen un carácter cíclico y de modificación; lo cual genera que la planeación del emprendimiento empresarial no sean un proceso pasivo donde se localizan un conjunto organizado y complejo de potencialidades naturales, humanas e institucionales y de sistemas de asentamiento poblacional, sino también un espacio en el que convergen los intereses, la identidad y cultura de una comunidad (Ferrandis, 2017)

De tal manera que el emprendimiento no puede concebirse desde el concepto de oportunidad, ni desde los rasgos del individuo, ni desde sus capacidades para lograr la efectividad. Porque se debe comprender que la planeación empresarial se dinamiza de las funciones de la economía política, y se da apertura a la identificación de una nueva área praxeológica, epistemológica, axiológica y ontológica, desde la perspectiva antropológica, linguiística, biológica, y por qué no psicoanalítica, que estudia este tipo de relaciones y características y, por ende, su complejidad. (Rodríguez, 2009)

Lo cierto es que todas estas funciones de la economía política, tienen una estrecha relación con la práctica. Esta última función, promueve la base científica para la acción práctica, cuyo dinamismo en la gestión empresarial facilita al ser humano de herramientas e instrumentos que promuevan su supervivencia, a partir de continuo proceso, sistematizado que correlacione la planeación, la institucionalidad, el emprendimiento y la economía política (Álvarez, 2017)

\section{Metodología}

Metodológicamente, a partir del método teórico, se fundamenta los hechos y fenómenos de cada función de la economía política, permitiendo crear premisas para comprender la correlación con el emprendimiento empresarial.

Por tanto, es necesario entender los fundamentos teóricos del análisis de las funciones de la Economía Política en el emprendimiento empresarial. En el sentido amplio de la palabra es la ciencia de las leyes que rigen la producción y el intercambio de los medios materiales de vida en la sociedad humana la ciencia de las condiciones y las formas bajo las que producen y cambian lo producido las diversas sociedades humanas”. (Engels 1963:179)

La función cognoscitiva de la Economía Política, se orienta hacia el estudio de las relaciones sociales de producción que se establecen entre los hombres con motivo de la producción, unida orgánicamente con las restantes relaciones sociales y las fuerzas productivas; representa el sistema de conocimientos que sustenta la teoría de dicha ciencia.

En esta misma línea, la función metodológica, por su parte, está dirigida a servir de fundamento teórico del resto de las ciencias económicas, tanto de aquellas que explican los aspectos funcionales y sectoriales de la sociedad, como de ciencias económicas que se relacionan con otras ramas del conocimiento. 
Razón por la cual, la función cognoscitiva como la metodológica no pueden ser analizadas de manera indistinta a la función práctica ya que esta refleja las acciones prácticas de la sociedad en el proceso de transformaciones económicas, sociales y políticas. Por tanto, todo proceso metodológico de emprendimiento empresarial, fundamenta su interpretación de los problemas contemporáneos a partir de la función ideológica de la Economía Política.

\section{Resultados}

\section{La función cognitiva de la Economía Política y el emprendimiento empresarial}

En un análisis cronológico, la función cognitiva ha dado paso en el siglo XXI a lo que se denomina "capital cognitivo", la base de la sociedad del conocimiento, el cual se crea independientemente del sistema político, social y productivo, pues la ciencia y el conocimiento que dentro de ella se genera, pertenece a ella misma. Podemos plantear que esta teoría se sustenta en la nueva Economía Política presentada por algunos autores, en lo que se conoce como el capitalismo cognitivo. (Boisier, 2013), (Correa, 2012)

Este último conduce a un desarrollo del capital basado en el conocimiento derivado de la fuerza colectiva y el trabajo cooperativo. Es una nueva fase que se apoya en la exploración sistemática de la información, la competencia de conocimiento de diversas tecnologías y las capacidades institucionales, asociados a numerosas formas de implicación en redes (Boisier, 2013). Este tipo de trabajo inmaterial es beneficioso en el proceso productivo pues enriquece las formas de transformación de las materias primas en mercancía, implementando novedosas técnicas y tecnologías. (Lazarrato, 2013)

En contrapartida, existe también el término de socialismo cognitivo (Ossa, 2012; Fonseca, 1996), considerado una evolución y actualización del socialismo científico creado por Marx y Engles en el siglo XIX, en base al análisis crítico y científico del capitalismo Este socialismo aboga por un conocimiento a la disposición de todos, donde no existen dueños, ni propiedad intelectual, pues ella hace que el conocimiento se convierta en propiedad privada; donde se requiere de saberes con una libertad de conciencia y los trabajadores puedan acceder a las ideas que desarrollan sin que esto genere conflictos en sí se viola o no la libertad de expresión y el querer saber.

Ecuador, ha establecido leyes en los últimos años para transformar su matriz productiva y convertirla principalmente en una economía social del conocimiento, una vertiente comercial del socialismo cognitivo, como una transición del capital cognitivo al socialismo cognitivo, es poner el conocimiento al acceso de todos para tener una sociedad cada vez más capacitada, pero al mismo tiempo es convertir a la ciencia en mercancía de un alto valor. Pero ella por sí sola no resulta comercialmente atractiva, por lo que debe asociarse al proceso productivo, ya sea de bienes o servicios. Retornando al tema del capital cognitivo, en este sentido una de las mayores interrogantes es ¿cómo convertir trabajo inmaterial en mercancía cognitiva 
(Ejemplo: ¿software, técnicas de dirección, modelos de gestión), que transforme su valor en valor de cambio y uso? ¿Cómo se convierte el conocimiento como medio de producción? Esta temática genera discusiones en todos sus ámbitos sobre todo entre los científicos se considera que el conocimiento tal cual otras mercancías poseen un valor de uso y un valor de cambio, es el resultado de un trabajo.

Aunque el trabajo científico teórico y aplicado, por lo general pueda ser autónomo de la producción esto no excluye que los sistemas productivos formados por campesinos, artesanos, constructores entre otros, sean la inspiración de la producción científica y su campo de acción práctica.

Muchos generadores de capital cognitivo menosprecian en términos científicos el empirismo, solo porque estas personas quizás no estén tan actualizadas en consideración con los adelantos de la ciencia y la tecnología. Sin embargo, en estos sistemas de producción, encontramos emprendedores empíricos con un alto grado de conocimiento teórico del sector donde se desarrollan, o viceversa, emprendedores exitosos en la gestión productiva territorial con escasos estudios.

En sentido general, todo el conocimiento necesario para el desarrollo de relaciones productivas da paso a innovaciones, mejores relaciones de competitividad, reducir la incertidumbre en los fenómenos económicos, asumir riesgos con un mayor nivel de información, mejorar las condiciones de planeación empresarial, conduce estos términos a una expresión que representa el flujo necesario del conocimiento de forma colectiva denominándolo "sinergía cognitiva" ideado para funcionar en el ámbito mesoeconómico y mesopolítico regional. Este capital cognitivo, necesario en las relaciones de producción, se vincula estrechamente con el denominado capital social dentro del cual se establece el aprendizaje individual y colectivo (Engels, 1878)

En la Teoría marxista, el capital en conjunto como trabajo producen plusvalía, lo que hace posible su reproducción y acumulación, Es decir, esta forma específica de capital social reside en las relaciones de producción. Durston (2001) define el capital social como el contenido de ciertas relaciones sociales, las que combinan actitudes de confianza con conductas de reciprocidad y cooperación que proporcionan mayor beneficio. el capital social como el contenido de ciertas relaciones sociales, las que combinan actitudes de confianza con conductas de reciprocidad y cooperación que proporcionan mayor beneficio.

Entre las variables que conforman el capital social en función de esas categorías fundamentales, encontramos la confianza interpersonal, así como, la cooperación de redes, importantes para las pequeñas y medianas empresas; base económica fundamental para la planeación empresarial. Esto nos permite establecer una premisa de investigación, tal cual se expresa en la Figura 1: 


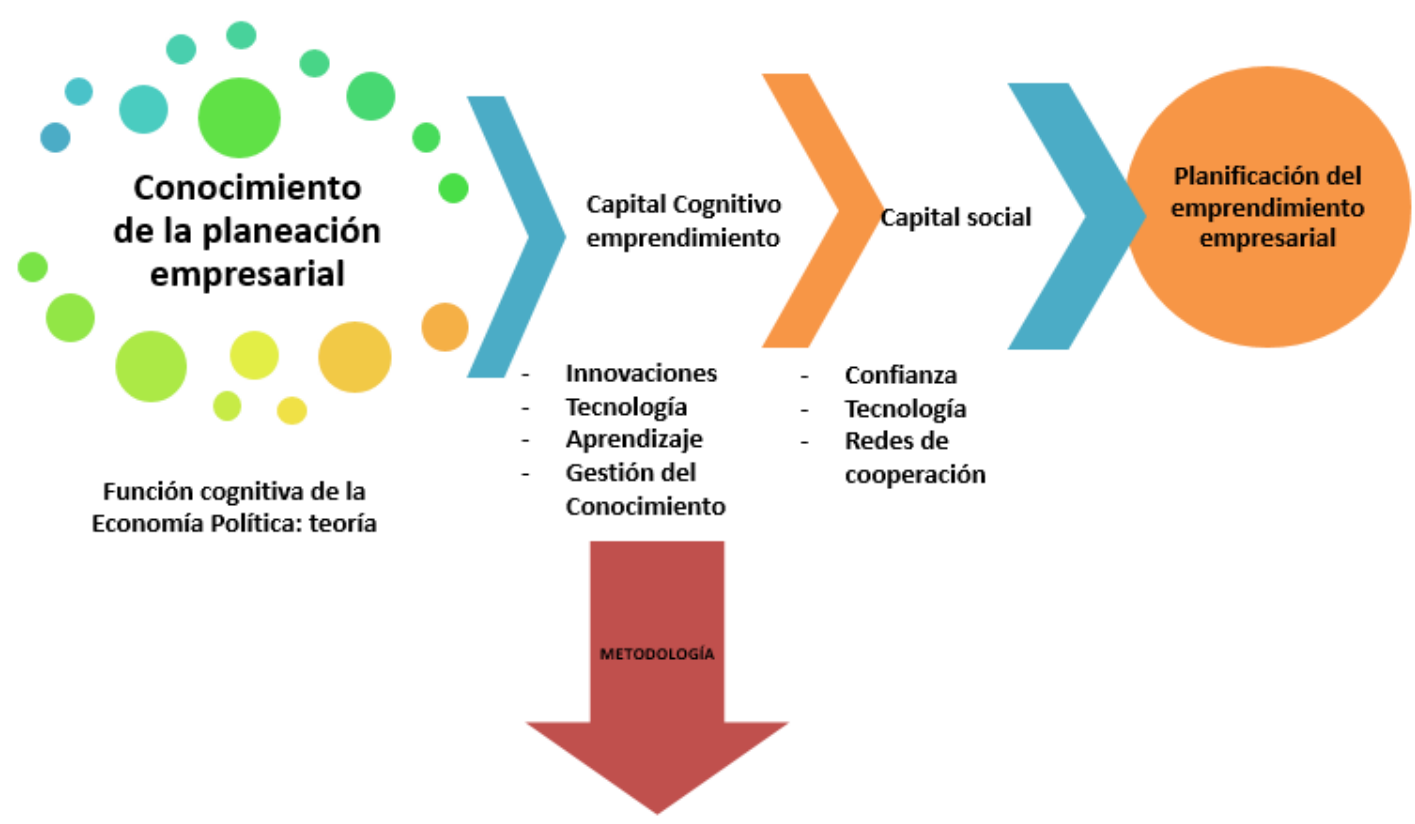

Figura 1. Premisa de investigación P1.

Fuente: Elaborado por los autores

P1. La función cognitiva de la Economía Política, formada por el conocimiento de la planeación empresarial del sistema productivo sostenible a través del capital cognitivo del emprendimiento y social impacta positivamente la planificación del emprendimiento.

En el criterio de Picchio (2005) los vacíos cognitivos en la gestión productiva desde la óptica de la Economía Política podrían estar determinados también por contradicciones de género. En algunos países latinoamericanos el trabajo productivo es discriminatorio por género desde la remuneración hasta la producción en sí, e incluso amparados por leyes que establecen que el salario de mujeres que ocupan los mismos puestos que los hombres sea menor, o aquellas que estereotipan las producciones por género o edad.

En otro orden de análisis, la carencia de recursos materiales, tecnológicos, el desconocimiento del mercado, provoca que el oferente sea quien determine los requerimientos del bien o servicio, entrando en contradicción con la ciencia, la cual plantea que la rentabilidad está en la medida en que la organización logre resultados favorables sobre la base de la satisfacción plena de las necesidades del cliente, como elemento de la sociedad.

El conocimiento científico del mercado, su estudio, la determinación de sus actitudes, percepciones, deseos, poder adquisitivo y otros factores de segmentación, su comportamiento de compra y su demanda, permiten llevar a cabo el proceso de planificación para establecer proporciones necesarias entre la producción y el consumo, lo cual es un factor importante para el emprendimiento. En este sentido el objeto sintetizado de la Economía Política, el consumo impulsa la producción y es el mercado quien condiciona la realización o no del 
producto, quien está dispuesto a pagar un precio determinado por acceder a ese beneficio, al producto genérico que para él tiene un valor de uso, como se muestra Figura 2.

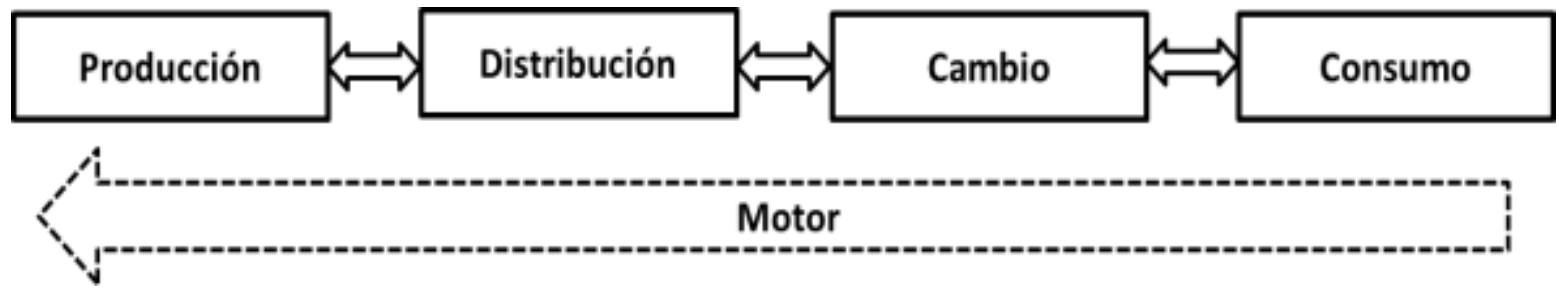

Figura 2

Objeto de la Economía Política Fuente: Elaborado a partir de Marx, s/f.

En tal sentido la postura del bagaje cognitivo también necesita de retroalimentación, evolución, de herramientas y mecanismos que permitan su continuidad; un ejemplo es la innovación que, como resultado esencial del aprendizaje, necesita ser creciente, por lo que requiere de una metodología. Es en este punto donde la función cognitiva se conecta con la función metodológica.

2. La función metodológica de la Economía Política y la planeación del emprendimiento empresarial

La función metodológica de la Economía Política, interviene en la planeación del emprendimiento empresarial explicando la base, esencia, contradicciones y leyes que regulan los procesos económicos cuyas formas externas investigan las demás ciencias económicas. Esta surge para responder cuestiones tales como: ¿qué métodos utilizar, ¿cómo establecerlo en los distintos sistemas productivos, qué adaptaciones realizar al conocimiento para que sea aplicable a cada emprendimiento, ¿cómo aplicar la perspectiva histórica-crítica del método marxista en la gestión territorial productiva sostenible?

Específicamente, en este estudio, no se pretende determinar el peso relativo de cada método, sino más bien describir cuales pueden ser aplicables al objeto de estudio y como estos contribuyen a exponer con mayor claridad como los elementos políticos, sociales e ideológicos permiten comprender mejor las características particulares de la gestión territorial productiva.

En la planeación empresarial, uno de los factores impositivos importantes a tratar es la equidad tributaria, los ingresos monetarios en el acceso al bienestar y como los ingresos tributarios tienen el rol e importancia de ser el instrumento redistribuidor del ingreso. La equidad impositiva podría ayudar en la sostenibilidad de los modelos, siguiendo la premisa universal de Marx que a cada quien según su capacidad; aquellos que más riquezas generen dada su capacidad, conocimiento y trabajo, también deberán ser los que más tributen. Esa necesidad de los métodos nos ayuda a inducir una segunda premisa, tal como se muestra en la Figura 3. 


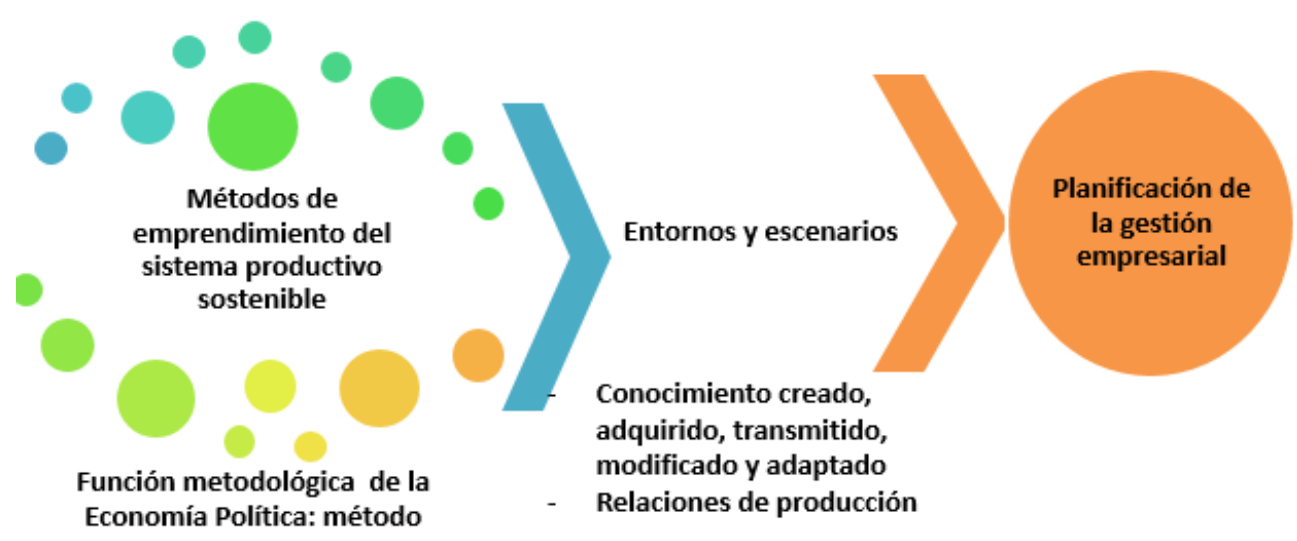

Figura 3. Premisa de investigación P2.

Fuente: Elaborado por los autores

P2. La función metodológica de la Economía Política permite el dominio de elementos conceptuales y metodológicos de la planificación del emprendimiento empresarial Según Fonseca (2016): "La planificación del emprendiendo empresarial depende de su habilidad para estimular iniciativas locales, generar nuevas empresas y llevar una dinámica de innovación productiva. La sostenibilidad de los emprendimientos empresariales debe ser abordada desde un enfoque multidimensional y sistémico que considere aspectos bióticos y abióticos, sociales, culturales, así como las modalidades de gestión de los territorios y su gobernabilidad"

Es por ello, que la metodología que se desarrolle en este ámbito debe tomar en cuenta el sistema productivo a partir de la planeación del emprendimiento desde todos sus componentes y relaciones. Cuando se promueve un emprendimiento, es importante saber que piensa la comunidad local, de lo contrario no sería posible construir un desarrollo sostenible. La inclusión como método, puede ser un principio básico en la planificación del emprendimiento empresarial. Conocer los criterios de esa Sociedad o comunidad.

\section{La función ideológica de la Economía Política y la planificación de la gestión territorial}

Cuando nos centramos en los entornos y escenarios en los cuales se desarrollará la ideología, vemos que pueden surgir políticas que faciliten o limiten el proceso productivo estudiado. Algunos clásicos consideran que es necesario completar la obra iniciada por Marx 
examinando la estructura del sistema de representaciones en el conjunto de la sociedad, para de esta forma estudiar la relación de las ideologías con el conocimiento (Castro, 2006)

La función ideológica establece que los individuos se unan bajo un pensamiento común de forma voluntaria, no, para determinar sus prácticas sociales, económicas y políticas, de forma coherente y con mayor sentido. Es la forma en que se interpretan las relaciones, la concepción del mundo; por lo general establecen normas morales y pueden ser asimiladas inconscientemente.

La ideología que sigue una la planeación del emprendimiento, puede definirle el horizonte en la toma de decisiones para resolver sus conflictos. Es por ello que podemos plantear como tercera premisa de esta investigación, tal como se muestra en la Figura 4.

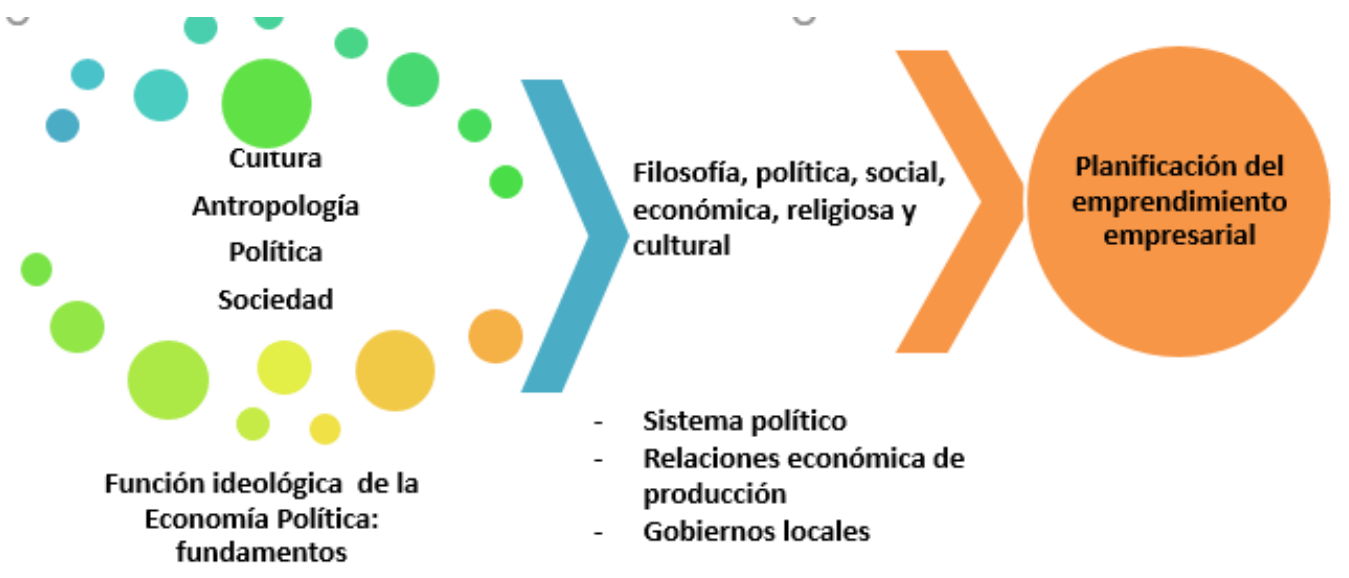

Figura 4. Premisa de investigación P3.

Fuente: Elaborado por los autores

P3. La función ideológica de la Economía Política permite identificar el papel que desempeñan los aspectos culturales e ideológicos como elementos condicionantes de la planificación del emprendimiento empresarial.

Para ello, deberá concebirse a la Economía Política como una ciencia en desarrollo continuo y que debe nutrirse, no sólo de las ideas de los clásicos del marxismo-leninismo, sino también del análisis crítico del pensamiento económico universal en su evolución hasta nuestros días; así como de la sistematización teórica y política en torno a las realidades económicas nacionales contemporánea

Las relaciones de propiedad sobre los medios de producción condicionan la distribución y el intercambio que encuentran su expresión en la Ley económica fundamental de cada modo de producción reflejando las relaciones económicas entre los hombres, la producción y el modo de lograrlo.

En el capitalismo, esos medios de producción tienen un carácter privado, perteneciendo al capitalista, el no siempre coincide con el productor. En el socialismo los medios de producción son propiedad social y el objetivo directo de la producción social resulta la satisfacción de las demandas de la sociedad, buscando mejoras en su calidad de vida. 
Siguiendo estas reflexiones, tendríamos también que preguntarnos de cómo lograr reducir el riesgo mediante la planificación del emprendimiento empresarial, si estos en alguna medida fuera en contra de sus ideologías. Un aspecto importante es recogido en el pensamiento de Castro-Gómez (2000): “...las ideologías son capaces de dotar a los hombres de normas, principios y formas de conducta, pero no de conocimientos sobre la realidad. La ideología no nos dice qué son las cosas sino cómo posicionarnos frente a ellas y, desde este punto de vista, no proporciona "conocimientos" sino únicamente "saberes" ...los hombres no la conocen, la viven."

Es por esta razón que se requiere de una función práctica que logre establecer los elementos de la realidad en los saberes de cada uno de sus contextos.

\section{La función práctica de la Economía Política y la planeación del emprendimiento empresarial}

Percibimos como en la función práctica reaparece el capital social con un conocimiento empírico que puede ser a su vez científicamente argumentado. Las tendencias internacionales más actuales del desarrollo territorial están encaminadas a minimizar sus impactos negativos ambientales y socio- culturales.

El objetivo primordial es satisfacer las necesidades del territorio, que el uso productivo de la tierra y la exportación de los recursos esté en consonancia con la conservación de la naturaleza y con culturas desconocidas evitando la perdida de identidades, seleccionando aquellos productos que respetan al medio ambiente natural, cultural y social. La gestión territorial productivo sostenible constituirá siempre el objetivo todo sistema de producción ya sea en los sectores priorizados o terciarios. constituyéndose su clave de éxito o fracaso la actividad humana transformadora y en la unidad de la reflexión.

En ese sentido, la cuarta premisa de esta investigación, tal cual se expresa en la Figura 5.

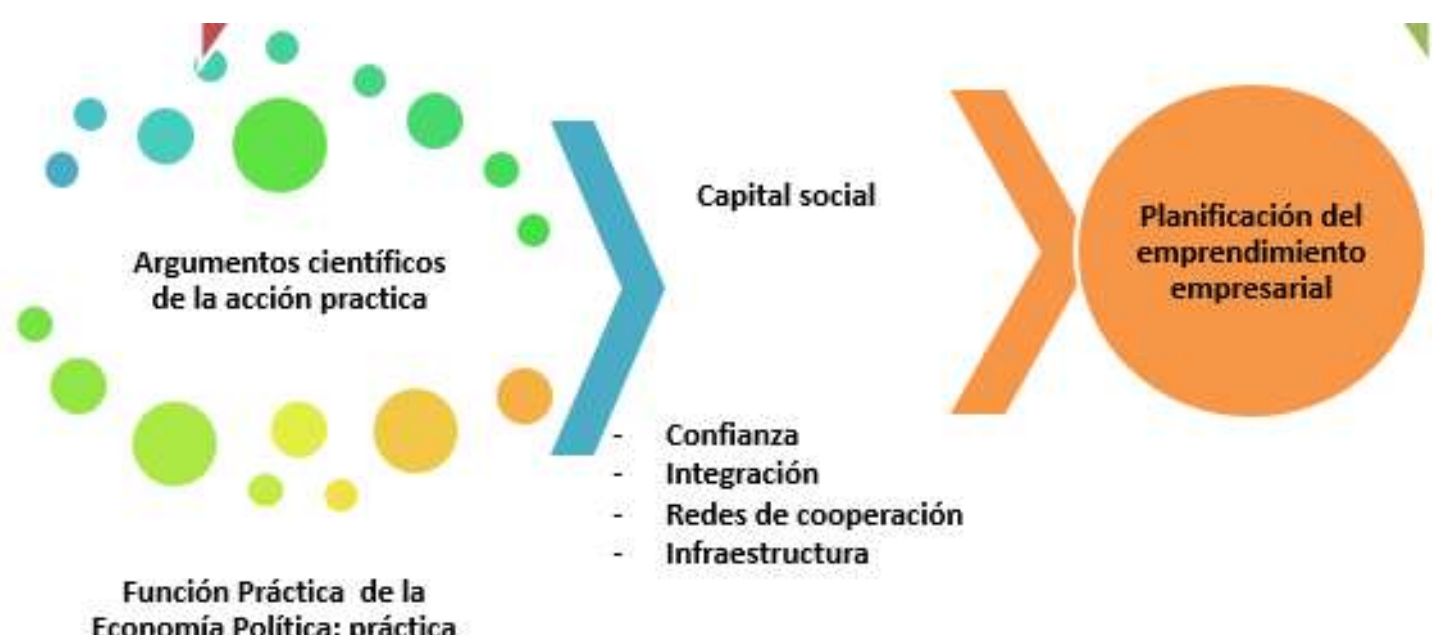

Figura 5. Premisa de investigación P4.

Fuente: Elaborado por los autores 
A partir del año 2007 hasta la actualidad, se promueve en el Ecuador un nuevo modelo de gestión para el desarrollo, el cual se denomina Plan Nacional para el Buen Vivir (PNBV) y consta de tres fases: 1) PNBV (2007 - 2009), 2) PNBV (2009 - 2013), 3) PNBV (2013 2017) y PNBV (2017 -2021) planes de desarrollo a escala nacional que han evolucionado y mejorado, se enfocan en garantizar una efectiva gestión socio productivo; a partir del cambio la matriz productiva. (SEMPLADES, 2018)

En este ámbito según estudio realizado por la Espae Graduate School of Management de la Espol, publicado en la Revista Lideres, el 22,7\% de los adultos afirmó haber emprendido aprovechando una oportunidad en el mercado, mientras que el 12,1\% lo hizo por necesidad. Con esas cifras, Ecuador pasó a tener la tasa de emprendimientos por necesidad más alta de la región, seguido de Perú (5,25\%) y Brasil (4,95\%). Según analistas consultados, el emprendimiento por necesidad está vinculado con los índices de desempleo $(5,58 \%$ hasta marzo) y subempleo (44,25\% hasta marzo). Más allá de las cifras, que pueden resultar distantes y frías, el ecuatoriano que emprende está consciente de las dificultades que existen para montar y sostener un negocio

Pero, el cantón Baños de Agua Santa de la provincia del Tungurahua, caracterizado por ser uno de los principales destinos turísticos del Ecuador; no tiene un modelo de gestión territorial validado para lograr desarrollo sostenible, el cual le permita mitigar los problemas y perdidas producto de la falta de sistematización entre las cadenas productivas, distribución y redistribución acorde al territorio y en base a sus necesidades; problemática emergente que surge porque se pierde de vista la disyuntiva entre territorio y sostenibilidad, siendo primordial el conocimiento de los preceptos teóricos de la Economía Política la cual tiene como misión solucionar los fenómenos económicos a la par de conservar, desarrollar los rasgos y la aplicación de medidas económicas.

En este ámbito, de las diversas problemáticas que enfrenta el emprendedor identificar un modelo de negocio realizable, rentable y sostenible, por lo cual resulta útil resolver el siguiente problema. ¿Qué vamos a ofrecer?, ¿Cuánto y porqué van a pagar nuestros futuros clientes por lo que les ofrecemos? ¿Cuántos clientes necesitamos para poder rentabilizar las inversiones realizadas y que el negocio se sostenga en el tiempo?, ¿Quién conforma el equipo? ¿De qué se ocupa cada uno? ¿Qué cualidades aporta y por qué él y no otra persona se va a ocupar de ello? ¿Cubrimos con este grupo todas las necesidades del negocio? Cuestionamientos que ayudan a determinar la sostenibilidad del emprendimiento.

La realidad que enfrenta el emprendedor es que la práctica es mucho más dura que la teoría y si no lo asumimos o no estamos preparados para ello nuestro negocio fracasará; con lo cual, se demuestra la necesidad de aplicación de las funciones de la Economía Política en las relaciones sociales de producción, porque la planeación del emprendimiento empresarial 
basas en sesgos; no le permite al empresario o joven visionario del mercado, alcanzar la sistematización teórica de la realidad económica donde se desarrolla con la práctica; lo cual genera que no mejoren las condiciones de vida de todos los participantes del emprendiendo puesto en marcha, sobre la base de una producción eficiente y sostenible.

En este sentido, para lograr la planeación del emprendimiento empresarial de forma sostenida se requiere:

- $\quad$ Es posible cumplir la premisa 1 planteada siempre y cuando se logre recopilar y retroalimentar el emprendedor de datos sobre sus realidades, para así evitar propuestas que lejos de ayudar degraden su visión entrono a la realidad

- $\quad$ La premisa 2 planteada se cumple una vez que se determine a través de una comparación inicial, cuáles son los métodos actuales que posee el empresario o joven visionario, cuáles son los métodos y modelos contemporáneos y cómo adaptarlos a la realidad ecuatoriana en cada territorio en busca de ser productivosostenible.

- $\quad$ Para el cumplimiento de la premisa 3, es necesario establecer un diagnóstico del desempeño de los aspectos culturales e ideológicos como elementos condicionantes de la planificación estratégica y las finanzas, utilizando quizás métodos y herramientas de la economía, finanzas, administración, sociología y la antropología.

- $\quad$ Finalmente, la premisa 4, establecida para la función práctica de la Economía Política se debe contextualizar la productividad, identificando y calculando sus indicadores, el uso de los factores productivos y así poderlos integrar a un proceso de planeación del emprendimiento empresarial sostenido.

\section{Conclusiones}

- Las funciones de la Economía Política en el emprendimiento empresarial, fomenta la redistribución equitativa de los beneficios económicos, así como el respeto de la cultura, el ambiente y sus valores, radicando su importancia en las categorías y el método científico para su desarrollo.

- $\quad$ El conocimiento del proceso de planeación del emprendimiento empresarial ayuda a lograr la obtención de negocios sostenible, enriqueciendo a su vez la función cognitiva de la Economía Política, lo cual permite comprobar el impacto de la planificación del emprendimiento en el éxito empresarial.

- Metodológicamente las funciones de la Economía Política, fomentan el análisis dinámico del sector empresarial, el uso de los factores y sistemas productivos, la reorganización espacial y descentralización de las relaciones funcionales, así como la generación de coopetencia.

- La Economía Política como ciencia, a partir de sus funciones coadyuva para que la tendencia ideológica de desarrollo siempre considere hechos históricos, 
fomentando que los nuevos lineamientos en la praxis promuevan la sostenibilidad de un negocio, donde el hombre sea el principio y el fin de toda actividad.

\section{Referencias bibliográficas.}

Álvarez, R. (2017). El territorio como factor del desarrollo. Revista Semestre Económico. [en línea] 13(27), 39- 62, 2010 [Consulta: 27 de mayo de 2019]. Disponible en: http://www.redalyc.org/pdf/1650/165016946002.pdf

Boisier, S. (2013) Sociedad del conocimiento, conocimiento social. Revista Internacional de Desenvolvimiento Local. Interações, 2(9-28), 2001. ISSN 0873-6529

Castro, A. (2006) Los estudios culturales y el concepto de ideología. Revista Iberoamericana, 66 (155 - 193) 2006. ISSN 0034-9631

Correa, H. (2012). Capitalismo cognitivo: problemas y desafíos para la economía política, Ed. Universitaria de Buenos Aires, Argentina, 2012. 145 p.

Durston, J. (2011) División del Desarrollo Social: capital social. Ed. Legis, 374p. ISBN: 958-653-0858-X

Engels, F. (1878) La revolución de la ciencia de Eugenio Dühring ("anti-dühring").

Editorial Política Habana, 1878. 230 p.

Ferrandis A. (2017) A propósito del desarrollo sostenible: origen, evolución y teorías alternativas. Revista de desarrollo local. [en línea] 2 (1): 74-96., 2016. [Consulta: 27 de mayo de 2019]. Disponible en: https://ojs.uv.es/index.php/TERRA/article/view/8216

Fonseca, J (2016) Novos paradigmas para uma nova sociedade: socialismo cognitivo, demosofia e existência cultural. Revista A Rede Faro, Associação In Loco. 31 (507 - 527). ISSN 0873-4205

García M. (2012) Consideraciones acerca de la función metodológica de la Economía Política en la formación de economistas en Cuba. Ponencia presentada al panel Retos de la Teoría Económica Contemporánea en los marcos del Evento XL Aniversario de los Estudios de Economía, efectuado en la Universidad de La Habana, (Habana). 20p.

Izerrougene. B (2011) Economía Política do Cognitivo. Revista Economía. Brasilia (DF), 2 (411-432). 2008. ISSN 0873-4625

Lazzarato, M. (2013) Immaterial Labor. Ed. University of Minnesota Press, Minneapolis. 189 p. ISBN 2162-2078

Macías X (2009). La gestión territorial como instrumento para el desarrollo rural. Ed.

Estudios Agrarios. 80p ISBN: 978-81-89578-22-5

Marx, k. (s/f) El Capital. Editorial Ciencias Sociales. Ed. Instituto Cubano del Libro, La Habana. Tres Tomos. s/f.

OSSA. C. (2012) Política y desarrollo sostenible. Revista Observatorio Cultural .8 (9 - 16). ISSN 2154-4794 
Picchio, Antonella (2005). La Economía Política y la investigación sobre las condiciones de vida. Por una economía sobre la vida. Aportaciones desde un enfoque feminista. Revista de Economía Política 8 (15 - 42). 2005. ISSN 5754-4358

SEMPLADES. Plan Nacional del Buen Vivir 2013 - 2017. 99- 150, 2013 [Consulta: 27 de mayo de 2019]. Disponible en: http://www.buenvivir.gob.ec/versiones-plan-nacional\#tabs3

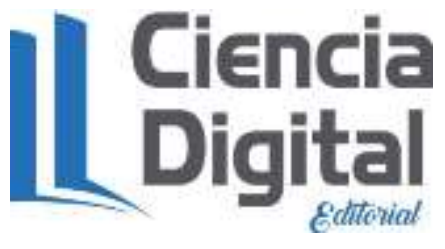




\section{PARA CITAR EL ARTÍCULO INDEXADO.}

Fonseca Vásconez, J., Villacis Venegas, N., Toledo Castillo, N. del R., Torres Guananga, G., \& Salazar Tenelanda, M. (2019). La planeación del emprendimiento empresarial. Un enfoque a partir de la $\begin{array}{lllll}\text { economía política. } & \text { Ciencia } & \text { Digital, } & \text { 440-445. }\end{array}$ https://doi.org/10.33262/cienciadigital.v3i2.6.576

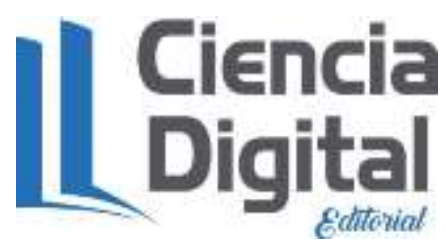

El artículo que se publica es de exclusiva responsabilidad de los autores y no necesariamente reflejan el pensamiento de la Revista Ciencia Digital.

El artículo queda en propiedad de la revista y, por tanto, su publicación parcial y/o total en otro medio tiene que ser autorizado por el director de la Revista Ciencia Digital.
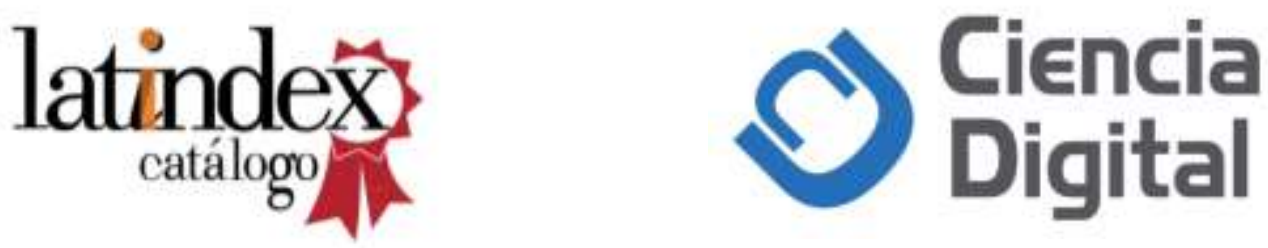